\title{
Prevalência de helmintos em patos domésticos Cairina moschata dom. (Linné) (Anseriformes, Anatidae, Cairinini, Cairina) provenientes de criações extensivas no estado do Rio de Janeiro, Brasil
}

\section{Prevalence of helmintos in domestic ducks Cairina moschata dom. (Linné) (Asncriformes, Anatidae, Cairinini, Cairina) proceeding from extensive creations in the state of Rio de Janeiro, Brazil}

\author{
Dalton Garcia de Mattos Junior, ${ }^{*}$ Daisy de Araújo da Costa, ${ }^{* *}$ Rodrigo Caldas Menezes, ${ }^{\star \star *}$ Eliana Marques Mesquitaa ${ }^{\star \star * *}$
}

\section{Resumo}

De março a agosto de 2002 foi realizado um levantamento de parasitos em trinta patos domésticos criados extensivamente oriundos de seis municípios do estado do Rio de Janeiro, através de exames clínicos, necropsias e amostras de tecidos afetados. Dezessete aves estavam parasitadas por oito diferentes espécies de helmintos. A prevalência e a intensidade média de infecção entre as aves parasitadas foram registradas. O presente trabalho registra, pela primeira vez no Brasil, nematóides da espécie Capillaria phasianina nestes hospedeiros.

Palavras-chave: Cairina moschata, helmintos, prevalência, Brasil.

\begin{abstract}
From March to August 2002 a survey concerning the parasites of the muscovy ducks (Cairina moschata dom.) was carried out. These waterfowls were originated from six counties of Rio de Janeiro state and were bred in free-range system. This research was based in clinic examination, necropsies and tissue samples collection for histopathology of 30 waterfowls. Birds were classified in to two-aged groups: young ones $(3-5$ months, $n=10)$ and adult ones $(5,5-36$ months, $n=20)$. Seventeen ducks were found parasitized by eight different helminth species. The prevalence and means intensities of infection among parasitized birds are given. In the present work the nematode Capillaria phasianina was reported for the first time as a new host for waterfowls (Cairina moschata dom.) in Brazil.
\end{abstract}

Keywords: Cairina moschata, helminths, prevalence, Brazil.

\section{Introdução}

Os patos domésticos são aves rústicas que se adaptam bem ao cativeiro, em local fresco com boa disponibilidade de água, e são criados em total liberdade nos sítios, chácaras, fazendas ou quintais. Essas aves são utilizadas como fonte alternativa de carne e ovos, ainda restritamente em nosso país. É uma criação de fácil manejo e baixo custo que produz carne macia e de boa qualidade. Estes anatídeos são descendentes da espécie selvagem sul-americana Cairina moschata, que tem ampla distribuição geográfica, ocorrendo desde o México até o norte da Argentina. No Brasil, a espécie selvagem só é encontrada no Pantanal mato grossense e na região Sul e é conhecida popularmente pelos nomes: "pato-asa-branca", "patobravo", "pato-picaço", "pato-selvagem". (Coimbra Filho, 1965).
No Brasil, existem poucas referências bibliográficas sobre a ocorrência e prevalência de helmintos nestas aves. O presente trabalho tem como objetivo realizar um levantamento das espécies de helmintos nesses hospedeiros criados extensivamente, distribuídos em seis municípios do estado do Rio de Janeiro, verificando a prevalência e a intensidade média de infecção desses helmintos em uma população de aves escolhidas ao acaso.

\section{Material e métodos}

No período de março a agosto de 2002, 30 patos domésticos foram adquiridos a partir de seis criações extensivas de subsistência, uma em cada um dos seguintes municípios do estado do Rio de Janeiro: Cachoeiras de Macacu, Niterói,

\footnotetext{
Prof. da disciplina Doenças Parasitárias dos Animais Domésticos, do Departamento de Saúde Coletiva Veterinária e Saúde Pública, MSV da Faculdade de Veterinária da Universidade Federal Fluminense, UFF - daltonmattos@ig.com.br.

** Médica-veterinária autônoma, MSc em Medicina Veterinária, Ornitopatologia.

*** Médico-veterinário, Centro de Criação de Animais de Laboratório - CECAL, do Instituto Oswaldo Cruz - Fiocruz - Rio de Janeiro - RJ.

${ }^{* * * *}$ Professora do Departamento de Tecnologia de Produtos de Origem Animal, MTA da Faculdade de Veterinária da Universidade Federal Fluminense, UFF.
} 
Nova Iguaçu, Rio Claro, São João de Meriti, Seropédica. As aves foram escolhidas aleatoriamente, de ambos os sexos e com faixa etária variando de 3 a 36 meses, divididas em dois grupos: 10 aves jovens de 3 a 5 meses e 20 adultos de 5,5 a 6 meses.

As aves eram, então, encaminhadas ao Laboratório de Doenças Parasitárias dos Animais Domésticos da Faculdade de Veterinária da Universidade Federal Fluminense, examinadas e, posteriormente, submetidas à eutanásia, de acordo com o preconizado pela ASGAV (2007).

Após a necropsia de cada ave, os órgãos dos sistemas respiratório, digestório e urinário e todos os seus conteúdos foram examinados com o auxílio de um microscópio estereoscópico, de acordo com a técnica preconizada por BIRGEL et al. (1979).

Os helmintos encontrados foram coletados, contados e preparados de acordo com as técnicas usuais em helmintologia após serem fixados em álcool - formol - ácido acético (AFA). Os cestóides e trematódeos foram corados pela solução bórax-carmim e montados em bálsamo do Canadá, entre lâminas e lamínulas, sendo identificados segundo Wardle e Mc Leod (1952), Travassos (1965), Khalil (1994) e Travassos et al. (1969) e Thatcher (1993), respectivamente. Os nematóides coletados foram contados e fixados, clareados pelo lactofenol e montados em bálsamo do Canadá, sendo identificados de acordo com Yamaguti (1961), Levine (1980) e Vicente et al. (1995). Os espécimens foram depositados na Coleção Helmintológica do Instituto Oswaldo Cruz, registrados com as seguintes identificações: CHIOC 35030 e CHIOC 35038 para os cestóides, CHIOC 35033 a-b para trematódeos e CHIOC 35028 a-c, CHIOC 35029, CHIOC, CHIOC 35031 a-b, CHIOC 35032 a-b, CHIOC 35034 a-c, CHIOC 35039 a-b, CHIOC 35035, CHIOC 35036, CHIOC 35037 a-b, CHIOC 35049 a-b para nematóides.

Fragmentos de órgãos com alterações macroscópicas associadas à presença dos helmintos foram coletados e fixados em formol 10\%, processados em parafina, cortados e corados pelo método da hematoxilina-eosina e montados entre lâminas e lamínulas, para o estudo histopatológico segundo Behmer et al. (1976).

\section{Resultados e discussão}

O Quadro 1 mostra as espécies identificadas de helmintos e os respectivos habitats. Da população de 30 aves estudadas, $56,6 \%(17 / 30)$ apresentavam-se parasitadas por uma ou mais espécies de helmintos. A prevalência de nematóides foi de $56,6 \%(17 / 30)$, dos cestóides 6,6\% (2/30) e dos trematódeos $3,3 \%(1 / 30)$.
No Quadro 2 estão demonstradas as freqüências das infecções isoladas e múltiplas, onde se observa que $10 \%$ das aves se encontravam parasitadas por um gênero de helmintos, $26,6 \%$ (8) por dois gêneros de helmintos, 20\% (6) por três gêneros de helmintos. Nos casos de infecção múltipla, as associações mais freqüentes foram Hadjelia neglecta e Capillaria sp. (83,3\%), e Hadjelia neglecta e Capillaria phasianina (73,3\%).

\begin{tabular}{|l|c|c|c|}
\hline Helmintos & Prevalência & $\begin{array}{c}\text { Intensidade } \\
\text { média de } \\
\text { infecção entre } \\
\text { os parasitados }\end{array}$ & $\begin{array}{c}\text { Amplitude } \\
\text { de variação }\end{array}$ \\
\hline Hadjelia neglecta & $53,3 \%$ & 7 & $1-114$ \\
Capillaria sp. & $30 \%$ & 10 & $1-89$ \\
Capillaria phasianina & $20 \%$ & 18 & $2-107$ \\
Tetrameres fissispina & $13,3 \%$ & 4 & $1-15$ \\
Eucoleus cairinae & $6,6 \%$ & 4 & $2-7$ \\
Lateriporus sp. & $3,3 \%$ & 2 & 2 \\
F. fasciolaris & $3,3 \%$ & 24 & 24 \\
E. revolutum & $3,3 \%$ & 2 & 2 \\
\hline
\end{tabular}

Quadro 2: Cairina moschata dom., prevalência, intensidade média de infecção e amplitude de variação das infecções de helmintos, no Estado do Rio de Janeiro no período de março a agosto de 2002.

A prevalência de $56,6 \%$ de helmintos em patos domésticos se aproxima aos registros de Pereira e Amato (1998) em Anas bahamensis (Linné) e Amazonetta brasiliensis (Gmelin), que apresentaram prevalências de helmintos de $55,6 \%$ e $50 \%$ respectivamente, em aves naturais do Brasil. Altas prevalências foram observadas em países da Europa e América do Norte (Delacour,1964; Reid e Mc Dougald, 1997).

No presente estudo, os nematóides foram os mais prevalentes, seguidos dos cestóides e trematódeos.

Quanto à intensidade média de infecção entre as aves parasitadas, o cestóide Fimbriaria fasciolaris foi o espécimen de maior quantidade de indivíduos coletados, dados esses também observados por Pereira e Amato (1998) em patos da espécie Anas bahamensis.

O número de aves fêmeas parasitadas foi bem maior do que os machos, e isto pode ser devido às altas exigências nutricionais impostas no período de síntese do ovo, fenômeno observado por Drobney e Fredrickson (1979).

O trematódeo Echinostoma revolutum foi encontrado na bolsa cloacal, mesmo habitat que Travassos (1965), Carvalho (1974) e Costa et al. (1986) registraram.

\begin{tabular}{|l|l|l|}
\hline \multicolumn{1}{|c|}{ Classe } & \multicolumn{1}{|c|}{ Espécies } & \multicolumn{1}{c|}{ Habitat } \\
\hline NEMATODA & $\begin{array}{l}\text { Capillaria phasianina Kotlán,1914 } \\
\text { Capillaria sp Pinto e Almeida, 1935 } \\
\text { Eucoleus cairinae (Freitas e Almeida, 1935) Lopez e Neyra, 19477 } \\
\text { Hadjelia neglecta (Lent e Freitas, 1939) Chabaud, 1975 } \\
\text { Tetrameres fissispina (Diesing, 1860) Travassos, 1914 }\end{array}$ & $\begin{array}{l}\text { esôfago e cecos } \\
\text { esôfago, cecos e vesícula biliar } \\
\text { esôfago } \\
\text { proventrículo, moela e cecos } \\
\text { proventrículo }\end{array}$ \\
\hline CESTODA & $\begin{array}{l}\text { Fimbriaria fasciolaris (Pallas,1781) Frolich, 1802 } \\
\text { Lateriporus sp Fhurmann, 1908 }\end{array}$ & $\begin{array}{l}\text { jejuno } \\
\text { jejuno }\end{array}$ \\
\hline TREMATODA & Echinostoma revolutum Frolich, 1802 & bolsa cloacal \\
\hline
\end{tabular}

Quadro 1: Cairina moschata dom., espécies de helmintos identificados de acordo com o habitat coletados através da necropsia, no período de março a agosto de 2002 
Seis espécies de helmintos identificadas aqui encontram-se relacionadas na lista de helmintos parasitas de animais domésticos do Brasil de acordo com Costa et al. (1986), mas Lateriporus sp. apenas foi registrado por Travassos (1965), da mesma forma que foi registrado neste estudo.

O presente trabalho registrou, pela primeira vez, Cairina moschata dom. como novo hospedeiro de Capillaria phasianina, parasitando: esôfago e cecos. Este nematóide foi registrado em faisões (Phasianus colchicus torquatus, Gmelin) parasitando intestinos, segundo Vicente et al. (1995).

Esses registros acrescentam informações sobre sete novas ocorrências de espécies de helmintos em patos no estado do Rio de Janeiro, complementando, assim, a lista de helmintos parasitas dos animais domésticos realizadas neste estado, feita por Duarte (1981) e Costa et al. (1986).

\section{Conclusões}

- Dos helmintos identificados, os nematóides apresentaram maior prevalência neste hospedeiro.

- Hadjelia negleta, nematóide de proventrículo, apresentou maior número de exemplares coletados.

- O pato doméstico é o novo hospedeiro de Capillaria phasianina no estado do Rio de Janeiro.

\section{Agradecimentos}

Agradecemos a colaboração do Prof. Jorge Bruno Nacinovic, da Seção de Ornitologia do Museu Nacional (UFRJ), pela orientação taxionômica e biológica da ave estudada a aos pesquisadores Joaquim Júlio Vicente e Luís Cláudio Muniz Pereira, do Setor de Helmintologia da Fiocruz, pela confirmação das espécies de helmintos encontrados.

\section{Referências}

ASGAV. Comitê técnico de Ovos da UBA Bem-Estar Animal na Produção de Poedeiras:regras e padroes recomendados.19 p. Disponível em:http://www.asgav.com.br/files/public/BEMESTAR\%20 POEDEIRAS\%20TEXTO.doc. Acessado em 5/06/2007.

BEHMER, O.A., TOLOSA, E.N.C.; FREITAS NETO,A.G. Manual técnico para histologia normal e patológica. São Paulo: EDART, 265 p. 1976.

BIRGEL, E. H.; NETTO, L. P.; AMARAL, V.; GIORGI, W.; PANETTA, J.C.; Meios e métodos de diagnóstico em Medicina Veterinária. 5. ed. Sociedade Paulista de Medicina Veterinária. Varela. 1979, 218 p.

CARVALHO, O.S. "Echinostoma revolutum"(Frolich, 1802) (Trematoda - Echinostomatidae) em pato doméstico de Minas Gerais. Atas da Sociedade de Biologia do Rio de Janeiro, v. 17, n. 2, p. 73-75, 1974.

COIMBRA FILHO, A.F. Apontamentos sobre "Cairina moschata" - (L., 1758) e seu hibridismo com "Anas platyrhynchos" (L., 1758) (Anatidae,Aves). Revista Brasileira de Biologia, Rio de Janeiro, v. 25 n. 4, p. 387-394, 1965.

COSTA, H.M.A. Distribuição de Helmintos Parasitos de Animais Domésticos no Brasil. Arquivo Brasileiro de Medicina Veterinária e Zootecnia, v. 38, n. 4, p. 465-579, 1986.

DELACOUR, J. The Waterfowl of the World. England: The Hamllym Publ. Group Ltd. v. IV. 1964, 388 p.

DROBNEY, R.D.; FREDERICKSON, L.H. Food selection by wood ducks in relation to breeding status. Journal of Wildlife Management, v. 43, p. 109-120, 1979.

DUARTE, M.J.F. Helmintos Parasitas dos Animais Domésticos no Estado do Rio de Janeiro. Arquivo Escola Veterinária UFMG, Belo Horizonte, v. 33, n.1, p. 67-98, 1981.
KHALIL, L. Keys to the Cestode Parasites of Vertebrates. Cambridge: CAB Internacional. 1994, 751 p.

LEVINE, N.D. Nematode Parasites of Domestics Animal and Man. Minn: Burguess Publ. Co. 1980, 477 p.

PEREIRA, L.C.M.; AMATO, S. B. Fimbriaria fasciolaris and Cloacotaenia megalops (Eucestoda-Hymenolepididae). Cestodes from Brazilian Waterfowl. Memórias Instituto Oswaldo Cruz. Rio de Janeiro, v. 93, n. 6, p. 767-772, 1998.

REID, W.M., MCDOUGALD, L.R. Cestodesand Trematodes. In: CALNEK, B.W. et al. Diseases of Poutry. 10. ed. USA: lowa State University Press. 1997, 929 p.

THATCHER, E.V. Trematódeos Neotropicais. Manaus. Instituto Nacional de Pesquisas da Amazônia. 1993, 553 p.

TRAVASSOS, L. P. Contribuição para o Inventário Crítico da Zoologia no Brasil - Fauna Helmintológica: Considerações Preliminares. Cestódeos. Rio de Janeiro: P.A. do Museu Nacional, v. 50, 109 p., 1965.

TRAVASSOS, L.P.; FREITAS, J.F.T.; KOHN, A. Trematódeos do Brasil. Memórias Instituto Oswaldo Cruz. Rio de Janeiro, v. 67, p. 1-886, 1969. VICENTE, J.J.; RODRIGUES, H.O.; GOMES, D.C.; PINTOR.M. Nematóides do Brasil. Parte IV. Nematóides de Aves. Revista Brasileira de Zoologia, v. 12, n.1, p. 273, 1995.

WARDLE, R.A.; MC LEOD, J.A. The Zoology of Tapeworns. Minneapolis: University of Minessota Press. 1952, 780 p.

YAMAGUTI, S. Systema Helminthum. The Nematodes of Vertebrates. Interscience, New York, v. 3, n.1/2, p. 1261, 1961. 